VIII.

\title{
Über die Funktion und die mikroskopische Anatomie des Gehörorgans bei totaler Aplasie der Schilddrüse.
}

Von

\author{
Prof. Siebenmann.
}

(Mit Tafel III.)

Ist es schon an und für sich auffällig, daß die mit Affektionen des inneren Ohres behafteten Kröpfigen nach unseren ausgedehnten Erfahrungen meistens dem männlichen Geschlechte angehören, während ja doch die Degeneration der Schilddrüse bäufiger bei den Frauen gefunden wird, so mub unser Glaube an die „dysthyre Schwerbörigkeit" vollenđs erschïttert werden angesichts der Tatsache, dah viele Menschen trotz ihrem gewaltigen Kropfe ein durchwegs normales Gehör besitzen. In dieser Hinsicht war folgender Fall für uns besonders beweisend, weil die ausgedehnte kropfige Degeneration, resp. die Kleinheit der noch funktionsfäbigen Drüsensubstanz hier operativ festgestellt worden war und sie im Laufe der folgenden drei Jahre zu strumipriver Kachexie, aber nicht zur geringsten Hörstörung gefüht hatte.

Hönicke, Ernst, 13 jährig, trat im Juni 1905 in unsere klinische Abteilung wegen bedentender inspiratorischer Dyspnoe. Er war vor 3 Jahren wegen beidseitigem grobem Kropf und stenotischen Erscheinungen auswärts operiert worden. Nach schriftlicher Mitteilung des Spitalarztes war die eine Seite der Schilddrüse total, die andere bis a uf einen ganz kleinen Rest degeneriert. Letzterer wurde bei der Operation geschont, scheint aber durch eine sich anschließende tiefe Abscedierung nachtrăg . lich auch noch zugrunde gegangen zu sein. Beim Spitalaustritt, welcher einige Wochen später erfolgte, war die Dyspnoe bedeutend gesteigert, und es entwickelte sich nun rasch das ganz charakteristische Bild der strumipriven Kachexie: Auffallende Abnahme der geistigen und körperlichen Regsamkeit sowie der Intelligenz, Appetitlosigkeit und Anämie, Stillstand des Wachstums, gelbliches trockenes, welkes Aussehen der Haut, Ausfallen und Struppigwerden der Kopfhaare. Röntgenuntersuchungen ergaben die charakteristischen Wachstumshemmungen der Epiphysengegendea. Zudem besteht - wahrscheinlig infolge der überstandenen Phlegmone - beiderseits Posticuslähmung. - Auf der medizinischen Klinik (Prof. H i s) wurden später Stoffwechseluntersuchungen vorgenommen und auf der chirurgischen Klinik (Prof, Enderlen) Implantationen von Schilddrüsenstücken versucht. -

Das Gehor war durchaus normal. Die Hörweite betrug für Flüstersprache (Zahlen) rechts mindestens 18 Meter, links 22 Meter. 
Noch wichtiger als die Untersuchungsresultate an bloß klinisch und operativ beobachteten Kröpfigen oder Athyreotischen sind natirlich die Ergebnisse, welche bei solchen athyreotischen Individuen gewonnen wurden, deren Schilddriisenlosigkeit duroh die Autopsie auf makroskopischem und mikrosk op is chem Wege festgestellt worden ist. Zu einer endgiltigen Entscheidung über diese Frage dürfte daher die folgende Abhandlung führen, welche auszugsweise in der otologischen Sektion der Naturforscherversammlung in Stuttgart mitgeteilt worden ist und einen Fall betrifft, bei welchem die obgenannten Anforderungen in vollständiger Weise erfüllt sind.

Sow eit dies durch die klinische Beobachtung festgestellt werden konnte, haben alle Individuen, bei denen der angeborene absolute Schilddrüsenmangel auch anatomisch, d. b. durch dic Autopsie nachgewiesen worden ist, ein bedeutend herabgesetztes Sprachvermögen; in der Regel sind sie aber nicht taub. Wie das Erkennen bestimmter Farben, so ist auch das Unterscheidungsvermögen für akustische Eindrücke bei der in solchen Fällen durchwegs beobachteten Herabsetzung der Intelligenz selbstverständlich wenig entwickelt. Zudem fehlt es an den betreffenden Begriffen und Vorstellungen sowie am Antrieb zum Sprechen. Ebenso unvollkommen sind alle irgendwie komplizierteren Bewegungen; sogar diejenigen des Essens sind meistens auffallend langsam und unzweckmäBig; unter den Athyreotischen gibt es solche, die nicht einmal ohne Hilfe ibre Speise zum Mund bringen können. Unter diesen Umständen erklärt sich die in Langsamkeit und Wortarmut bestehende Mangelhaftigkeit der Sprache nngezwungen aus der Idiotie, welche diesen kretinenhaften, myxödematösen Individuen eigentümlich ist.

Von angeborener Aplasie der Schilddrüse sind nur wenige Fälle bekannt, welche Personen betreffen, die eine längere Reibe von Jahren gelebt haben, und bei denen die Athyreose auch durch die Autopsie sowie durch die mikroskopisehe Untersuchung in unanfechtbarer Weise festgestellt werden konnte. Hierher gehören zunächst die Kretinen, über welche Poehl und Maresch berichten und welche nieht tanb waren.

Unter seinen interessanten sieben Beobachtungen von "Idiotie avee oachexie pachydermique", welche Bourneville (Arch. de Neurologie 1886. Bd. XII. S. 137 und Bd. XVI, S. 97) in klinischer und pathologisch-anatomischer Beziehung genau untersucht 
Über die Funktion a. d. mikroskopische Anatomie đ. Gebörorgans usw. 85

und beschrieben hat, wurde totaler Mangel der Schilddrüse, aber in keinem Falle Taubheit konstatiert. Es findet sich darunter ein 15 jähriger Knabe, welcher sogar beim gewöhnlichen Primarunterricht lesen, schreiben und etwas rechnen lernte, und beim Spitaleintritt $z w e i$ Briefe schrieb, die man ihm diktierte; doch ist dies allerdings eine seltene Ausnahme, und ich führe sie nur an als Beweis für unsere Behauptung, daß mit angeborener Athyreosis zum mindesten kein höherer Grad von Schwerhörigkeit notwendig verbunden sein $m u B$.

In Übereinstimmang damit steht auch die vom nämliehen Autor konstatierte Tatsache, daß trotz sehr mangelhafter Sprache bei solchen Athyreotischen beide Schläfenlappen normal gebaut und sogar voluminös entwickelt sein können.

Wie verhält es sich nun mit der Anatomie des Ohres bei dem angeborenen totalen Fehlen der Sehilddritse? Die bis jetzt in der Literatur niedergelegten Befunde uber das Kretinenohr geben auf diese Frage keinen bestimmten Bescheid, da in den betreffenden Fällen die Untersuchung der Schilddrüse nicht in Betracht gezogen oder nur ungenügend ausgeführt worden ist. In den am besten beobachteten Fällen von Habermann aber handelte es sich um zwei Kretinen, deren Schilddrüse mit ibren Adnexen, wie aus dem zugehörenden, an anderer Stelle ausführlicher publizierten allgemeinen Sektionsbefund und der mikroskopischen Untersuchung (siehe Scholz, Untersuchnngen über den Kretinismus, Berlin 1906) hervorgeht, in beiden Fällen nicht wesentlich alteriert war: In dem einen Falle wird das Drisengewebe als "gut entwickelt", in dem anderen als "gehörig beschaffen und entsprechend funktionierend" bezeichnet. Die von $\mathrm{H}$ abermann beschriebenen Kretinenlabyrinthe stammen somit von Individuen, welche sicher nicht zur thyreopriven Gruppe (Athyreoidose Scholz) der Kretinen, sondern sehr wahrscheinlich, wie diejenigen von Moos und Steinbrïgge, zur Chondrodystrophie (Kaufmann) zu reehnen sind.

Wir wissen somit bis jetzt über den anatomischen Bau der Gehörorgane des Athyreotischen gar nichts; es ist dieser Mangel um so mehr zu bedauern, als gerade hier sich der von anderer Seite behauptete EinfluB der Schilddrüse auf das innere Ohr, falls ein solcher iberhaupt existiert, in der intensivsten Weise geltend machen müßte.

Ich bin nun heute in der glüeklichen Lage, Labyrinth- 
präparate beschreiben zu können von einem 41/2 Monate alten Kinde, welehes an Myxödem litt und im Kinderspital Basel kachektiseh zugrunde ging; bei demselben hat eine minutiöse makroskopische und mikroskopische Untersuehung nicht die Spur einer Schilddrise aber auch keine Veränderungen am häutigen Labyrinth noch am Hörnerv nachweisen können. Der Fall hat eine eingehende Bearbeitung gefunden durch Dr. Th. Dieterle, welcher bei Prof. Hagenbach im Baseler Kinderspital die klinische $B$ Beobachtung und bei Prof. Ka afmann an unserem pathologischen Institut die betreffeuden Untersuchungen gemacht hat. Seine Resultate sind teils in Virchows Archiv, Bd. CLXXXIV, S. 56, teils (während der Drucklegung dieser Arbeit) im Jabrbuch für Kinderheilkunde, Bd. LXIV niedergelegt. Diesen Ferren verdanken wir auch das seltene Präparat, welches den Gegenstand dieser Arbeit (resp. Vortrages) bildet.

Das uns zur mikroskopischen Untersuchung übergebene Felsenbein war in 4 proz. Formollösung fixiert worden; bei der Isolierung des Labyrinthwirfels und bei der Entkalkung fiel die elfenbeinerne Konsistenz des Knochens auf: die Decalcinierung des kleinen Präparates in 6 proz. Salpetersäure dauerte volle 14 Tage. Makroskopisch schien jedes spongiöse Gewebe in der Umgebung der Labyrinthkapsel zu feblen. Die weitere Behandlung und Einbettung geschah nach den Angaben von Siebenmann (vgl. Mittelohr und Labyrinth in Bardelebens Handbuch der Anatomie und Grundzige der Anatomie und Pathogenese der Taubstummheit 1904). Die Zerlegung erfolgte in Serienschnitten vertikal zur Längsachse der Felsenbeinpyramide, ungefäbr entsprechend der Ebene des oberen Bogenganges.

Trommelfell, Mittelohr und Tube sind normal. An der abgeblaßten und gehärteten Mittelohrschleimhaut fällt makroskopisch nichts besonders auf, während mikroskopiseh sich das Vorhandensein einer katarrhalisehen Veränderung ergibt. Das Epithel ist iberall erhalten, aber stark aufgelockert, entzindlich geschwellt, die Submucosa bedeutend verdickt, gefäb- und blutreich, ziemlich infiltriert mit mononucleären Leukocyten. Infolge der Schleimhautsehwellung sind die Nischen der Paukenhöhlenwände - besonders der ovalen Fensternische - fast ganz ausgefüllt. Es fehlt aber eine größere Exsudatansammlung. Dieser Befund entspricht einer Otitis media eatarrhalis a den letzten Lebenstagen, namentlich bei Kindern mit allgemein daniederliegendem Kräftezustand, aufzutreten ptlegt. 
Die Gestalt und Ausbildung der Räume des knöebernen Labyrinths weisen keine wesentlichen Abweichungen auf. Die Größenverhältnisse und die gegenseitige Lagebeziehung der einzelnen knöehernen Labyrinthteile entsprechen der Norm, namentlich gilt dies auch von den Fenstern, der Konfiguration der Spindel und den Sehneckenwindungen.

Das häutige Labyrinth weicht nicht von der Norm ab. Infolge später Sektion ist die Fixation der epithelialen Gebilde nicht ganz vollkommen, doch ist die Kernfärbung durchaus gut und die Strukturverhältnisse sind an den meisten Stellen wohl zu erkennen. Maculae und Cristae wie auch der Vorhofapparat überhaupt gut entwiekelt, die Nervenversorgung reichlich, Otolithenmembran nicht deutlich. Der Ductus cochlearis ist normal weit, ReiBnersche Membran iiberall gut erhalten, Limbus spiralis und Membrana tectoria sehr gut fixiert, letztere deutlich längsgestreift. - Wo das Corti sche Organ erhalten ist, zeigt es keine deutliche Abweichung in Größe und Gestalt seiner Zellen. An den meisten Stellen ist es aber aufgelockert und durch die herabgefallene Cortische Membran zerdrückt, so dab die einzelnen Zellen abgelöst und mit dem Striaepithel als Trümmer im Ductus cochlearis herumsehwimmen. Die nervösen Elemente zeigen qualitativ und quantitativ keine Abweichung, die Nervenkanäle sind überall gut gefüllt mit Nervenfasern; die Ganglienzellen haben das gewöhnliche Aussehen, das Volumen des Acustico facialis entspricht dem des normalen Neugeborenen.

Die mikroskopische Untersuchung ergibt somit normale Formen und Größenverhältnisse des Laby. rinths und seines Inhaltes. Es fehlen alle Veränderungen, die eine wesentliche Funktionsunfähigkeit des Gehörorgans bedingen könnten.

Auch die Labyrinthkapsel zeigt, wie eine Vergleichung mit verschiedenen normalen Präparaten gleichaltriger Individuen ergibt, einen normalen Knochenbau. Dagegen enthalten die großen, spaltförmigen, konzentrisch angeordneten Markräume 1) abnormerweise fast gar keine lymphoiden Zellen, sondern ein lockeres Fasermark, hier und da auch Fettmark (vgl. Taf. III). Der mit Eosin-Hämatoxylin sich hellrot färbende, die Labyrinthkapsel umhtillende, periostal gebildete Knochen besitzt die charakteristische schwere, dichte Struktur des Skelettes athyreotiseher Individuen;

1) Vergl. Siebenmann, Corrosionsanatomie des knöchernen Labyrinthes. S. $13-17$ und Fig. $3-4$. 
sein mikroskopisches Bild erinnert durchaus an dasjenige, welohes Dieterle von einem Quersehnitt dureh die Mitte der Femurdiaplyyse des nämlichen Individuums in der oben zitierten Arbeit Virch. Arch. Bd. CLXXXIV Taf. III, Fig. 3 gibt. -

In den Haversschen Kanälen finden sich auch hier nur wenige enge Gefäße und kelnarmes, lockeres, stellenweise mit Fettzellen durchsetztes Fasermark. Die Lamellen sind auffallend konzentrisch und dicht um die Jabyrinthkapsel angeordnet. Ein Verwisehen dieser fötalen Struktur, wie es im extrauterinen Leben durch Resorption und Apposition um die Haveissehen Kanäle stattfindet, hat sich nooh nirgends geltend gemacht. Auffallend ist auch der Umstand, daß die Wand der Haversseben Kanäle sich nicht so blänt, wie es beim normalen, mit Hämatoxylin-Eosin gefärbten Präparaten in diesem Entwicklungsstadium wenn auch nicht immer, so doch meistenteils der Fall ist. In abnormer Weise unverkalkt gebliebene Knorpelreste, wie sie Moos und Steinbrügge sowie Habermann im kretinischen Felsenbein antrafen, finden sich nirgends. -

Die Tatsache, daß die Schilddrüse für die Entwicklung des häutigen Labyrinthes während des intrauterinen Lebens entbehrlich ist, steht somit, wie Dieterle mit Recht betont, duroh diesen mitgeteilten Befund nun auch anatomisob begründet fest.

Die klinisohe Beobachtung aber, wonach selbst totaler Schilddrüsenmangel $a \mathrm{ch}$ im späteren $L$ e ben weder Taubheit noch hochgradige Schwerhörigkeit bedingt, sollte uns veranlassen, die Angaben der Myxödemkommission, welche ich dem Kapitel über die endemische Taubstummheit (vergl. Siebenmann, Anatomie und Pathogenese der Taubstummheit, 1904) zugrunde gelegt habe, beztiglich ihrer Daten über die Häufigkeit der Schwerhörigkeit bei Myxödem einer erneuten sorgfältigen Nachprüfung durch exfahrene Ohrenärzte zu unterziehen. Daß es viele dem myxödematösen athyreotischen Typus angehörende $\mathrm{Kr}$ etinen gibt, welche das Bild der nelvösen Schwerlo örigkeit bieten, steht sicher fest. Diese Form ron kretinischer Sehwerhörigkeit wird aber - wegen der Idiotie dieser Individuen - bezüglich der Häufigkeit ihres Vorkommens sicher bedeutend ubersebätzt. Sie findet sich zudem mindestens in der nämlichen Hâufigkeit aueh bei Kretinen anderer Gruppen, wie z. B. beim rhachitischen Zwergwuobs und bei der Chondrodyso trophie; dafir sprechen unter anderem die in unserer Fach- 
literatur vorliegenden mikroskopisehen Labyrinthuntersuchungen von schwerhörigen Kretinen, indem gerade die Athyreose nicht, die Chondrodystrophie aber überwiegend darunter vertreten zu sein seheint. - Was sehlieblich die das Ohr betreffenden Sektionsbefunde schwerhöriger, resp. tauber idioter Kretinen von $\mathrm{Nièpce}$ anbelangt, so sind sie derart, daß sie nach unseren heutigen Kenntnissen sicher auf überstandene meningitische Erkrankung zurückgeführt werden müssen. Denn es handelt sich meistens um Knochenauffillungen im Labyrinth und um entsprechende Hirnveränderungen, zum Teil auch um schon makroskopiseh erkennbare Sklerose des N. acusticus.

Die klinische Beobachtung, wonach selbst totaler Mangel der Sohilddrüse in der Regel nicht zu Schwerbörigkeit führt, muß uns daher ferner veranlassen, die "dysthyre Schwerhörigkeit $(\mathrm{B} 10 \mathrm{ch})^{\text {", }}$, welche nach diesem Autor auf kropfiger Degeneration oder Aplasie der Sohilddrüse beruben soll, auf andere Ursachen zurïckzuführen und eine solche Gruppierung zuriickzuweisen.

\section{Erklärung zu Tafel III.}

Das vorliegende mikroskopische Bild stellt ein Stück der äußeren Schneckenwand ron einem Kinde von $4^{1 / 2}$ Monaten dar. Dem Schneckenlumen zunächst liegt die mit Eosin-Hämatoxylin sich violett-blau fărbende Zone der Labyrinthkapsel, links davon schließt sich der rot tingierte Bindegewebsknochen der Pyramide an. Die Labyrinthkapsel enthält reichlich hellblau gefärbten Knorpel in Form von Interglobularräumen und typisch angeordnete große, gefäßführende, markarme Lymphräume - Verhältnisse, wie sie $a b$ und $z u$ auch bei normalen Individuen dieses Alters vorkommen. Dagegen ist der rote Bindegewebsknochen a ffallend dicht, sklerotis ch. 